\title{
Algebraic string bracket as a Poisson bracket
}

\author{
Hossein Abbaspour, Thomas Tradler and Mahmoud Zeinalian
}

\begin{abstract}
In this paper we construct a Lie algebra representation of the algebraic string bracket on negative cyclic cohomology of an associative algebra with appropriate duality. This is a generalized algebraic version of the main theorem of [AZ] which extends Goldman's results using string topology operations. The main result can be applied to the de Rham complex of a smooth manifold as well as to the Dolbeault resolution of the endomorphisms of a holomorphic bundle on a Calabi-Yau manifold.
\end{abstract}

Mathematics Subject Classification (2010). 55P35, 53D20, 14A22, 14A20.

Keywords. Free loop space, cyclic homology, symplectic reduction.

\section{Introduction}

Goldman's original work [Go] on the Lie algebra of free homotopy classes of oriented closed curves on an oriented surface was extensively generalized through the introduction of String Topology by Chas and Sullivan [CS]. In particular, they generalized this Lie bracket to one on the equivariant homology of the free loop space of a compact and oriented manifold $M$. From the beginning, it was clear that this bracket had a deep relation to the holonomy map on a vector bundle; see [Go], [CFP], [CCR], [CR]. This relation is the subject of a paper, [AZ], by the first and third author. It is shown there that using Chen's iterated integral one obtains a map of Lie algebras from the equivariant homology of the free loop space to the space of functions on a space of generalized flat connections.

Algebraic analogues of string topology Lie algebra have also been considered in recent years. Jones [J] had shown that for a simply connected topological space $X$ the equivariant homology of the free loop space is isomorphic to the negative cyclic cohomology of the algebra of cochains on $X$. Using this, and Connes' long exact sequence relating negative cyclic cohomology and Hochschild cohomology, together with the BV-algebra on Hochschild cohomology, Menichi [Men] deduced a Lie bracket on the negative cyclic cohomology in a way similar to the one in string topology [CS], Section 6.

The starting point for this work was to obtain a generalization of the results in [AZ] and place it in a more algebraic setting where the equivariant homology of the 
loop space is replaced by negative cyclic cohomology. A suitable setting for this is to consider a unital differential graded algebra $A$ over a field $k=\mathbb{R}$ or $\mathbb{C}$, with a reasonable trace $\operatorname{Tr}: A \rightarrow k$. Using the results of [T], the above assumptions imply an isomorphism $\mathrm{HH}^{*}(A, A) \cong \mathrm{HH}^{*}\left(A, A^{*}\right)$ of the Hochschild cohomologies of $A$ with values in $A$ and its dual $A^{*}$ such that the cup product on $\operatorname{HH}^{\circ}(A, A)$ and the dual of Connes' $B$-operator on $\mathrm{HH}^{\bullet}\left(A, A^{*}\right)$ make these spaces into a $\mathrm{BV}$-algebra. This BV-algebra, together with Connes' long exact sequence between the Hochschild cohomology $\mathrm{HH}^{\bullet}\left(A, A^{*}\right)$ and negative cyclic cohomology $\mathrm{HC}_{-}^{*}(A)$, imply a Lie algebra structure on $\mathrm{HC}_{-}^{*}(A)$ by a theorem of Menichi [Men], Proposition 7.1, which is based on a similar marking/erasing result of Chas and Sullivan [CS], Theorem 6.1.

Now, using work of Gan and Ginzburg in [GG], we may look at the moduli space

$$
\mathcal{M C}=\left\{a \in A^{\text {odd }} \mid d a+a \cdot a=0\right\} / \sim
$$

of Maurer-Cartan solutions. Since we only consider odd elements, the trace induces a symplectic structure $\omega$ on $\mathcal{M} \mathcal{C}$, and thus one can define a Poisson bracket on the function ring $\mathcal{O}(\mathcal{M C})$ of $\mathcal{M} \mathcal{C}$. More details of this construction will be given in Section 3.

We may connect the two sides of the above discussion via a canonical map $\left\{a \in A^{\text {odd }} \mid d a+a \cdot a=0\right\} \rightarrow \mathrm{HC}_{\cdot}^{-}(A), a \mapsto \sum_{n \geq 0} 1 \otimes a^{\otimes n}$, and dualizing this gives a map $\rho: \mathrm{HC}_{-}^{*}(A) \rightarrow \mathcal{O}(\mathcal{M} \mathcal{C})$. We may now compare the two Lie algebras from above. Our main result then states that the brackets are indeed preserved.

Theorem 1. $\rho: \operatorname{HC}_{-}^{2 \bullet}(A) \rightarrow \mathcal{O}(\mathcal{M} C)$ is a map of Lie algebras.

In a special case considered in [AZ] this map becomes the generalized holonomy map from the equivariant homology of the free loop space of $M$ to the space of functions on the moduli space of generalized flat connections on a vector bundle $E \rightarrow M$. In fact one has a commutative diagram

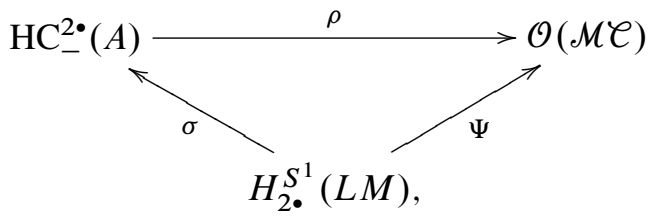

where $\Psi$ is the generalized holonomy discussed in [AZ] and $\sigma$ comes from Chen's iterated integral map, as described in Section 5. In particular, for $\operatorname{dim} M=2$, this recovers Goldman's results on the space of flat connections on a surface.

Another motivation for this work was the study of the algebraic structure of the deformation complex of a CY manifold. We were interested in the Hochschild and cyclic complexes of $A=\Omega^{0, *}(M, \operatorname{End}(E))$, the Dolbeault resolution of the endomorphisms of a strong generator $E$ of the category of perfect complexes on an algebraic CY manifold. The discussion of this paper, once done at the chain level, relates to the algebraic structure of the deformation complex. 
Finally, we remark that the above discussion generalizes in a straight forward way to the case of a cyclic $A_{\infty}$-algebra $A$. This will be the topic of the last Section 6. In fact, by the same reasoning as above, we obtain the Lie bracket on the negative cyclic cohomology $\mathrm{HC}_{-}^{*}(A)$. Also, by symmetrization we may associate an $L_{\infty}$-algebra to $A$, which induces a Maurer-Cartan space similar to (1). We find that the canonical map $\rho$ is still well defined such that Theorem 1 also remains valid in this generalized setting.

Notation. For a map $F$ of complexes, $F_{\bullet}\left(\right.$ resp. $\left.F^{*}\right)$ denotes the induced map in homology (resp. cohomology).

Acknowledgments. The authors would like to thank Victor Ginzburg and Luc Menichi for useful discussions and correspondence on this topic. The authors were partially supported by the Max-Planck Institute in Bonn and the second author warmly thanks the Laboratoire Jean Leray at the University of Nantes for their invitation through the Matpyl program.

\section{The Lie algebra $\mathrm{HC}_{-}^{\bullet}(A)$}

In this section, we recall the Lie algebra structure of the negative cyclic cohomology $\mathrm{HC}_{-}^{\bullet}(A)$, for a dga $(A, d, \cdot)$ with a trace $\operatorname{Tr}: A \rightarrow k$. The Lie bracket comes from the long exact sequence that relates negative cyclic (co-)homology to Hochschild (co-)homology. For simplicity, we will work in the normalized setting.

Definition 2. Let $\left(A=\bigoplus_{i \in \mathbb{Z}} A^{i}, d: A^{i} \rightarrow A^{i+1}, \cdot\right)$ be a differential graded associative algebra over a field $k$, and let $M=\bigoplus_{i \in \mathbb{Z}} M^{i}$ be a differential graded $A$-bimodule. The (normalized) Hochschild chain complex is defined as

$$
\bar{C}_{\bullet}(A, M):=\prod_{n \geq 0} M \otimes \bar{A}^{\otimes n},
$$

where $\bar{A}=A / k$ and $s$ denotes shifting down by one. The boundary $\delta: \bar{C}_{\bullet}(A, M) \rightarrow$ $\bar{C}_{\bullet+1}(A, M)$ is defined by,

$$
\begin{aligned}
\delta\left(a_{0} \otimes\right. & \left.a_{1} \otimes \cdots \otimes a_{n}\right) \\
:= & \sum_{i=0}^{n}(-1)^{\epsilon_{i}} a_{0} \otimes \cdots \otimes d\left(a_{i}\right) \otimes \cdots \otimes a_{n}+\sum_{i=0}^{n-1}(-1)^{\epsilon_{i}} a_{0} \otimes \cdots \\
\quad & \cdots \otimes\left(a_{i} \cdot a_{i+1}\right) \otimes \cdots \otimes a_{n}-(-1)^{\epsilon_{n}^{\prime}}\left(a_{n} \cdot a_{0}\right) \otimes a_{1} \otimes \cdots \otimes a_{n-1},
\end{aligned}
$$

where $a_{0} \in M, a_{1}, \ldots, a_{n} \in A, \epsilon_{0}=\left|a_{0}\right|, \epsilon_{i}=\left(\left|a_{0}\right|+\cdots+\left|a_{i-1}\right|+i-1\right)$, and $\epsilon_{n}^{\prime}=\left(\left|a_{n}\right|+1\right) \cdot\left(\left|a_{0}\right|+\cdots+\left|a_{n-1}\right|+n-1\right)$. Note that the differential is well defined; see [L]. Similarly, the (normalized) Hochschild cochain complex is defined by

$$
\bar{C}^{n}(A, M):=\left\{f: s \bar{A}^{\otimes n} \rightarrow M \mid f\left(a_{1} \otimes \cdots \otimes a_{i} \otimes \cdots \otimes a_{n}\right)=0 \text { if } a_{i}=1\right\} .
$$


Here the differential $\delta^{*}: \bar{C}^{\bullet}(A, M) \rightarrow \bar{C}^{\bullet-1}(A, M)$ is given by

$$
\begin{aligned}
\left(\delta^{*} f\right)\left(a_{1}\right. & \left.\otimes \cdots \otimes a_{n}\right) \\
:= & \sum_{i=1}^{n}(-1)^{|f|+\epsilon_{i}} f\left(a_{1} \otimes \cdots \otimes d a_{i} \otimes \cdots \otimes a_{n}\right)+d\left(f\left(a_{1} \otimes \cdots \otimes a_{n}\right)\right) \\
& +\sum_{i=1}^{n-1}(-1)^{|f|+\epsilon_{i}} f\left(a_{1} \otimes \cdots \otimes\left(a_{i} \cdot a_{i+1}\right) \otimes \cdots \otimes a_{n}\right) \\
& +(-1)^{|f|\left(\left|a_{1}\right|+1\right)} a_{1} \cdot f\left(a_{1} \otimes \cdots \otimes a_{n}\right) \\
& +(-1)^{|f|+\epsilon_{n}} f\left(a_{1} \otimes \cdots \otimes a_{n-1}\right) \cdot a_{n} .
\end{aligned}
$$

The respective (co-)homology theories are denoted by

$$
H_{\bullet}(A, M)=H\left(\bar{C}_{\bullet}(A, M), \delta\right), \quad \mathrm{HH}^{\bullet}(A, M)=H\left(\bar{C}^{\bullet}(A, M), \delta^{*}\right) .
$$

Denoting by $A^{*}=\operatorname{Hom}(A, k)$ the graded dual of $A$, we see that the dual of $\bar{C} \cdot(A, A)$ is given by $\bar{C}^{\bullet}\left(A, A^{*}\right)$. Recall furthermore that there is a cup product $\cup$ on $\bar{C} \bullet(A, A)$ defined by

$$
(f \cup g)\left(a_{1} \otimes \cdots \otimes a_{m+n}\right):=f\left(a_{1} \otimes \cdots \otimes a_{m}\right) \cdot g\left(a_{m+1} \otimes \cdots \otimes a_{m+n}\right) .
$$

Next, we define the (normalized) negative cyclic chains $\overline{\mathrm{CC}}_{\cdot}^{-}(A)$ of $A$ to be the vector space $\bar{C}_{\bullet}(A, A)[[u]]$, where $u$ is of degree +2 , and with differential $\delta+u B$, where $B: \bar{C}_{\bullet}(A, A) \rightarrow \bar{C}_{\bullet-1}(A, A)$ is Connes' operator,

$$
B\left(a_{0} \otimes a_{1} \otimes \cdots \otimes a_{n}\right):=\sum_{i=0}^{n}(-1)^{\epsilon_{i}} 1 \otimes a_{i} \otimes \cdots \otimes a_{n} \otimes a_{0} \otimes \cdots \otimes a_{i-1},
$$

where $\epsilon_{i}=\left(\left|a_{i}\right|+\cdots+\left|a_{n}\right|+n-i+1\right)\left(\left|a_{0}\right|+\cdots+\left|a_{i-1}\right|+i-1\right)$. Thus, every element of $\overline{\mathrm{CC}}_{n}^{-}(A)$ is an infinite sum $\sum_{i=0}^{\infty} a_{i} u^{i} \in \bar{C}_{\mathbf{\bullet}}(A, A)[[u]]$, where $a_{i} \in \bar{C}_{n-2 i}(A, A), \delta$ acts on $a_{i} \in \bar{C}_{\mathbf{0}}(A, A)$, and $u B$ acts as

$$
\ldots \stackrel{u B}{\longleftarrow} \bar{C}_{\bullet}(A, A) \cdot u^{2} \stackrel{u B}{\longleftarrow} \bar{C}_{\bullet}(A, A) \cdot u \stackrel{u B}{\longleftarrow} \bar{C}_{\bullet}(A, A) .
$$

Dually, define the (normalized) negative cyclic cochains $\overline{\mathrm{CC}}_{-}^{\bullet}(A)$ of $A$ by taking $\overline{\mathrm{CC}}_{-}^{\bullet}(A)=\bar{C}^{\bullet}\left(A, A^{*}\right) \otimes k\left[v, v^{-1}\right] / v k[v]$, where $v$ is an element of degree -2 . Explicitly, the degree $n$ part $\overline{\mathrm{CC}}_{-}^{n}(A)$ is represented by finite sums $\sum_{i=0}^{k} a_{i} v^{-i}$ where $a_{i} \in \bar{C}^{n-2 i}\left(A, A^{*}\right)$. The differential is given by $\delta^{*}+v B^{*}$, where $\delta^{*}$ acts on $\bar{C}^{\bullet}\left(A, A^{*}\right)$, and $v B^{*}$ acts as follows.

$$
\ldots \stackrel{v B^{*}}{\longrightarrow} \bar{C}^{\bullet}\left(A, A^{*}\right) \cdot v^{-2} \stackrel{v B^{*}}{\longrightarrow} \bar{C}^{\bullet}\left(A, A^{*}\right) \cdot v^{-1} \stackrel{v B^{*}}{\longrightarrow} \bar{C}^{\bullet}\left(A, A^{*}\right) .
$$

Note that if $C_{\mathbf{v}}(A, A)$ is finite dimensional in each degree, then the graded dual of $\overline{\mathrm{CC}}_{-}^{n}(A)$ is isomorphic to the chain complex $\overline{\mathrm{CC}}_{n}^{-}(A)=\operatorname{Hom}\left(\overline{\mathrm{CC}}_{-}^{n}(A), k\right)$; see also [HL], Lemma 3.7. It is easy to see that $B^{2}=\delta B+B \delta=0$, and we define the associated (co-)homology theories by

$$
\mathrm{HC}_{\bullet}^{-}(A)=H\left(\overline{\mathrm{CC}}_{\bullet}^{-}(A), \delta+u B\right), \quad \mathrm{HC}_{-}^{\bullet}(A)=H\left(\overline{\mathrm{CC}}_{-}^{\bullet}(A), \delta^{*}+v B^{*}\right) .
$$


Lemma 3. If $H_{\bullet}(A, A)$ is bounded from below, then both $\bar{C}_{\bullet}(A, A)[u]$ and $\bar{C}_{\bullet}(A, A)[[u]]$ with differential $\delta+u B$ calculate negative cyclic homology $\mathrm{HC}_{\bullet}^{-}(A)$.

This lemma follows from a spectral sequence argument for the inclusion $\bar{C}_{\text {. }}(A, A)[u] \hookrightarrow \bar{C}_{\text {. }}(A, A)[[u]]$, similarly to $[\mathrm{HL}]$, Lemma 3.6. Note that our sign convention is opposite to the one from [HL] but in agreement with [GJP] since our differential $\delta: \bar{C}_{\bullet}(A, A) \rightarrow \bar{C}_{\bullet+1}(A, A)$ is of degree +1 .

From now on, we additionally assume that we also have a suitable trace map.

Definition 4. Let $\operatorname{Tr}: A \rightarrow k$ be a trace map satisfying $\operatorname{Tr}(d a)=0$ and $\operatorname{Tr}(a b)=$ $-(-1)^{|a| \cdot|b|} \operatorname{Tr}(b a)$ for all $a, b \in A$. Assume furthermore that the map $\omega: A \rightarrow A^{*}$, $\omega(a)(b):=\operatorname{Tr}(a b)$, is a bimodule map which induces an isomorphism on homology $H(A) \rightarrow H\left(A^{*}\right)$. By abuse of language, we will also view $\omega$ as a map $\omega: A \otimes A \rightarrow k$, $\omega(a, b)=\operatorname{Tr}(a b)$. In this case, $A$ is also called a symmetric algebra.

Notice that $\omega: A \rightarrow A^{*}$ induces a morphism of the Hochschild complexes $\omega_{\sharp}: \bar{C} \bullet(A, A) \rightarrow \bar{C}^{\bullet}\left(A, A^{*}\right)$ via composition $\omega_{\sharp}(f):=\omega \circ f$, which is an isomorphism on homology $\omega_{\sharp}^{\bullet}: H^{\bullet}(A, A) \rightarrow H^{\bullet}\left(A, A^{*}\right)$. We may thus transfer the cup product $\cup$ on $H^{\bullet}(A, A)$ to a product $\sqcup$ on $\operatorname{HH}^{\bullet}\left(A, A^{*}\right)$ by setting $f \sqcup g:=$ $\omega_{\sharp}^{*}\left(\left(\omega_{\sharp}^{*}\right)^{-1} f \cup\left(\omega_{\sharp}^{*}\right)^{-1} g\right)$. Define furthermore the operator $\Delta: \mathrm{HH}^{*}\left(A, A^{*}\right) \rightarrow$ $\mathrm{HH}^{\bullet}\left(A, A^{*}\right)$ as the dual of $B$ on homology. Then we assume that $\left(\mathrm{HH}^{\bullet}\left(A, A^{*}\right), \sqcup, \Delta\right)$ is a $\mathrm{BV}$-algebra, i.e., $\sqcup$ is a graded associative, commutative product, $\Delta^{2}=0$, and the bracket $\{a, b\}:=(-1)^{|a|} \Delta(a \sqcup b)-(-1)^{|a|} \Delta(a) \sqcup b-a \sqcup \Delta(b)$ is a derivation in each variable.

Recall from Menichi [Men] that this BV-algebra induces a Lie algebra on the negative cyclic cohomology $\mathrm{HC}_{-}^{*}(A)$ using the long exact sequences of Hochschild and negative cyclic cohomology. The inclusion $\overline{\mathrm{CC}}_{\bullet}^{-}(A) \stackrel{\times u}{\rightarrow} \overline{\mathrm{CC}}_{\bullet}^{-}(A)$ given by multiplication by $u$ has cokernel $\bar{C}_{\bullet}(A, A)$. We thus obtain a short exact sequence

$$
0 \rightarrow \overline{\mathrm{CC}}_{\bullet}^{-}(A) \stackrel{\times u}{\longrightarrow} \overline{\mathrm{CC}}_{\bullet}^{-}(A) \rightarrow \bar{C}_{\bullet}(A, A) \rightarrow 0,
$$

which induces Connes' long exact sequence of homology groups:

$$
\cdots \rightarrow \mathrm{HH}_{n}(A, A) \stackrel{\mathfrak{B}_{\bullet}}{\longrightarrow} \mathrm{HC}_{n-1}^{-}(A) \rightarrow \mathrm{HC}_{n+1}^{-}(A) \stackrel{I_{\bullet}}{\rightarrow} \mathrm{HH}_{n+1}(A, A) \stackrel{\mathfrak{B}_{\bullet}}{\longrightarrow} \cdots
$$

Here the projection to the $u^{0}$ term $I: \overline{\mathrm{CC}}_{\bullet}^{-}(A) \rightarrow \bar{C}_{\bullet}(A, A)$ induces the map $I_{\bullet}$, and the connecting map $\mathcal{B}_{\bullet}$ is induced by the composition $\bar{C}_{\bullet}(A, A) \stackrel{B}{\rightarrow} \bar{C}_{\mathbf{0}}(A, A) \stackrel{\mathrm{inc}}{\longrightarrow}$ $\overline{\mathrm{CC}}_{\bullet}^{-}(A)$. Notice that unlike inc $\circ B: \bar{C}_{\bullet}(A, A) \rightarrow \overline{\mathrm{CC}}_{\bullet}^{-}(A)$, the inclusion inc: $\bar{C}_{\bullet}(A, A) \rightarrow \overline{\mathrm{CC}}_{\bullet}^{-}(A)$ is not a chain map.

Dually, we have the short exact sequence

$$
0 \rightarrow \bar{C}^{\bullet}(A, A) \rightarrow \overline{\mathrm{CC}}_{-}^{\bullet}(A) \rightarrow \overline{\mathrm{CC}}_{-}^{\bullet}(A) \rightarrow 0,
$$

inducing Connes' long exact sequence of cohomology groups

$$
\cdots \rightarrow \mathrm{HH}^{n}\left(A, A^{*}\right) \stackrel{I^{\bullet}}{\rightarrow} \mathrm{HC}_{-}^{n}(A) \mathrm{HC}_{-}^{n-2}(A) \stackrel{\mathcal{B}^{\bullet}}{\longrightarrow} \mathrm{HH}^{n-1}\left(A, A^{*}\right) \stackrel{I^{\bullet}}{\rightarrow} \cdots .
$$


Notice that the composition

$$
B^{\bullet}=\mathscr{B}^{\bullet} \circ I^{\bullet}
$$

is exactly the $\Delta$ operator of our $\mathrm{BV}$-algebra on $\mathrm{HH}^{\bullet}\left(A, A^{*}\right)$ so that we may obtain an induced Lie algebra from [Men], Lemma 7.2, much like the marking/erasing situation in $[\mathrm{CS}]$.

Proposition 5 (L. Menichi [Men]). The bracket $\{a, b\}:=I^{\bullet}\left(\mathscr{B}^{\bullet}(a) \sqcup \mathscr{B}^{\bullet}(b)\right)$ induces a Lie algebra structure on $\mathrm{HC}_{-}^{*}(A)$.

We end this section with some examples of the above definitions.

Examples 6. Let $M$ be a smooth, compact and oriented Riemannian manifold.

- A first example is obtained by taking $A=\Omega^{\bullet}(M)$ the de Rham forms on $M$, $d=d_{D R}$ the exterior derivative on $A$, and $\operatorname{Tr}(a):=\int_{M} a$.

- More generally, if $E \rightarrow M$ is a finite dimensional complex vector bundle over $M$, with a flat connection $\nabla$, then we may take $A=\Omega^{\bullet}(M, \operatorname{End}(E))$ with the usual differential $d_{\nabla}$. Similarly, the trace is given by a combination of integration and trace in $\operatorname{End}(E)$. The cyclic property of the trace guarantees that this induces an injective bimodule map $\omega: A \rightarrow A^{*}$ that is a quasi-isomorphism.

- Both of the above examples are special cases of elliptic Calabi-Yau space as defined in $[\mathrm{C}]$. By definition, this means that we have a bundle of finite dimensional associative $\mathbb{C}$ algebras over $M$, whose algebra of sections is denoted by $A$. Furthermore, there is a differential operator $d: A \rightarrow A$, which is an odd derivative with $d^{2}=0$ making $A$ into an elliptic complex, a $\mathbb{C}$ linear trace $\operatorname{Tr}: A \rightarrow \mathbb{C}$, a hermitian metric $A \otimes A \rightarrow \mathbb{C}$, and a complex antilinear, $C^{\infty}(M, \mathbb{R})$ linear operator $*: A \rightarrow A$ satisfying certain natural conditions. It can be seen that this example satisfies the above assumptions. The details and other examples of elliptic Calabi-Yau spaces can be found in $[\mathrm{C}]$ and $[\mathrm{DT}]$.

\section{Maurer-Cartan solutions}

In this section we define the moduli space of Maurer Cartan solutions for a symmetric algebra $A=\bigoplus_{i \geq 0} A^{i}$ and then explain its symplectic nature. The main reference for this section is the paper [GG] by Gan-Ginzburg together with Section 4 of [AZ]. Let us assume $k=\mathbb{R}$ or $\mathbb{C}$.

For $a, b \in A$ define the Lie bracket $[a, b]:=a \cdot b-(-1)^{|a| \cdot|b|} b \cdot a$ and the bilinear form $\omega(a, b):=\operatorname{Tr}(a b)$. The first remark is that $\left(A=A^{\text {odd }} \oplus A^{\text {even }}, d,[\cdot, \cdot], \omega\right)$ is a cyclic differential graded Lie algebra as it is defined in Section 4 of [AZ]. Therefore all results in [GG] apply here to define the Maurer-Cartan solutions. 
Definition 7. We define the Maurer-Cartan moduli stack as

$$
\begin{aligned}
M C & :=\left\{a \in A^{\text {odd }} \mid d a+\frac{1}{2}[a, a]=d a+a \cdot a=0\right\}, \\
\mathcal{M C} & :=M C / \sim,
\end{aligned}
$$

where the equivalence is generated by the infinitesimal action of $A^{0}$ on $A$. Here for $a \in A^{0}$, the vector field $\xi_{x}$ on $A$ is defined by

$$
\xi_{x}(a)=[x, a]-d x
$$

Recall that $\omega$ is a symplectic form and the infinitesimal action is Hamiltonian. Moreover, the map $\mu: a \mapsto \phi_{a} \in\left(A^{\text {even }}\right)^{*}$, where

$$
\phi(x)=\omega\left(d a+\frac{1}{2}[a, a], x\right)
$$

is the moment map corresponding to the Hamiltonian action above. One should think of the tangent space $T_{[a]} \mathcal{M} \mathcal{C}$ at a class $[a]$ as the 3 -term complex $T_{[a]} \mathcal{M} \mathcal{C}$ :

$$
T_{[a]}^{-1} \mathcal{M} \mathcal{M}:=A^{\text {even }} \stackrel{\xi(a)}{\longrightarrow} T_{[a]}^{0} \mathcal{M} \mathcal{M}:=T_{a} A^{\text {odd }}=A^{\text {odd }} \stackrel{\mu_{a}^{\prime}}{\longrightarrow} T_{[a]}^{1} \mathcal{M} \mathcal{C}:=A^{\text {even* }},
$$

graded by $-1,0$ and 1 . Here $\xi(a)$ is the map $x \mapsto \xi_{x}(a)$. The kernel of $\mu^{\prime}$ is the Zarisky tangent space to $M C$, and the image of $\xi(a)$ accounts for the tangent space of the action orbit. Ideally, when 0 is a regular value for $\mu$ and the infinitesimal action of $A^{\text {even }}$ on $M C=\mu^{-1}(0)$ is free, this complex is concentrated in degree zero, and the Zarisky tangent space to $\mathcal{M} \mathcal{C}$ at $[a]$ is the cohomology group $H^{0}\left(T_{[a]} \mathcal{M} \mathcal{C}\right)=$ $H^{*}\left(A^{\text {odd }}, d_{a}\right)$ where $d_{a} b=d b+[a, b]$.

Note that the 3 -term complex (11) is self-dual, where the self-duality at the middle term is given by the symplectic form

$$
\omega\left(X_{a}, Y_{a}\right):=\operatorname{Tr}\left(X_{a} \cdot Y_{a}\right) \in k .
$$

By assumption from the previous section, $\omega$ is non-degenerate. This gives rise to an isomorphism $T_{[a]} \mathcal{M} \mathcal{I} \stackrel{\simeq}{\longrightarrow}\left(T_{[a]} \mathcal{M} \mathcal{C}\right)^{*}$ and equips $\left(T_{[a]} \mathcal{M} \mathcal{C}\right)$ with a symplectic form given by (12). In the case of a nonsingular point $[a]$ this is the usual pairing on $H^{0}\left(T_{[a]} \mathcal{M} \mathcal{C}\right)=H\left(A^{\text {odd }}, d_{a}\right)$ induced by $\omega$.

The function space $\mathcal{O}(\mathcal{M} \mathcal{C})$ is defined to be the subspace of $\mathcal{O}(M C)$ invariant under the infinitesimal action. The symplectic form allows us to define the Hamiltonian vector field $X^{\psi}$ of a function $\psi \in \mathcal{O}(\mathcal{M C})$ via

$$
\omega\left(X_{a}^{\psi}, Y_{a}\right)=\mathrm{d} \psi_{a}\left(Y_{a}\right):=\lim _{t \rightarrow 0} \frac{d}{d t} \psi\left(a+t Y_{a}\right) \quad \text { for all } Y_{a} \in T_{[a]}^{1} \mathcal{M} \mathcal{C} .
$$

We then define the Poisson bracket on $\mathcal{O}(\mathcal{M C})$ by

$$
\{\psi, \chi\}:=\omega\left(X^{\psi}, X^{\chi}\right)=\operatorname{Tr}\left(X^{\psi} \cdot X^{\chi}\right) .
$$




\section{The induced Lie map}

In this section we define a map $\rho: \operatorname{HC}_{-}^{2 \bullet}(A) \rightarrow \mathcal{O}(\mathcal{M C})$ and prove that it respects the brackets. We start by defining a map $P: M C \rightarrow \bar{C} .(A, A)$, and in turn the map $R: M C \rightarrow \overline{\mathrm{CC}}_{\bullet}^{-}(A)$ which factors through $P$. Dualizing $R$ will induce the wanted map $\rho$.

Definition 8. Recall that $M C=\left\{a \in A^{\text {odd }} \mid d a+a \cdot a=0\right\}$ and $\bar{C}_{\cdot}(A, A)=$ $\prod_{n \geq 0} A \otimes \bar{A}^{\otimes n}$. Let then $P: M C \rightarrow \bar{C}_{\mathbf{c}}(A, A)$ be given by the expression

$$
P(a):=\sum_{i \geq 0} 1 \otimes a^{\otimes i}=(1 \otimes 1)+(1 \otimes a)+(1 \otimes a \otimes a)+\cdots .
$$

Note that $\delta(P(a))=\sum 1 \otimes a \otimes \cdots \otimes d a \otimes \cdots \otimes a+\sum 1 \otimes a \otimes \cdots \otimes(a \cdot a) \otimes \cdots \otimes a=0$ for $a \in M C$, due to the relation $d a+a \cdot a=0$ in $M C$. Thus, we obtain in fact a Hochschild homology class $[P(a)] \in \mathrm{HH}_{\bullet}(A, A)$.

Next define the map $R:=$ inc $\circ P$ as the composition $R: M C \stackrel{P}{\rightarrow} \bar{C}_{\bullet}(A, A) \stackrel{\text { inc }}{\longrightarrow}$ $\overline{\mathrm{CC}}_{.}^{-}(A)$. Just as above, we have that $\delta(R(a))=0$, and since we are in the normalized setting, we see that $B(R(a))=0$ and so $(\delta+u B)(R(a))=0$. The induced negative cyclic homology class is again denoted by $[R(a)] \in \mathrm{HC}^{-}(A)$. It is immediate to see that under the long exact sequence (8), we have that $I(R(a))=P(a)$.

Using the pairing between negative cyclic homology and negative cyclic cohomology, $\langle\cdot, \cdot\rangle: \mathrm{HC}_{-}^{\cdot}(A) \otimes \mathrm{HC}_{\bullet}^{-}(A) \rightarrow k$, we define the map $\rho$ by

$$
\rho: \mathrm{HC}_{-}^{\bullet}(A) \rightarrow \mathcal{O}(\mathcal{M} C), \quad \rho([\alpha])([a]):=\langle[\alpha],[R(a)]\rangle=\langle\alpha, R(a)\rangle,
$$

for $[\alpha] \in \mathrm{HC}_{-}^{*}(A),[a] \in \mathcal{M} \mathcal{C}$. To simplify notation, we will also write $\rho(\alpha)$ instead of $\rho([\alpha])$.

Lemma 9. $\rho$ is well defined.

Proof. We need to show that the value $\rho([\alpha])([a])=\langle\alpha, R(a)\rangle$ is independent of the choice of the representative $[a] \in\left\{x \in A^{\text {odd }} \mid d x+x \cdot x=0\right\} / \sim$. Infinitesimally, this amounts to showing that $L_{X(b)} \rho(\alpha)(a)=0$, where $L_{X(b)}$ is the Lie derivative along a vector field in the direction $X(b)_{a}=d b+[a, b] \in T_{[a]} M C$, for any $b \in A^{\text {even }}$. To see this, note that

$$
\begin{aligned}
L_{X(b)} \rho(\alpha)(a) & =\left(i_{X(b)} \circ d+d \circ i_{X(b)}\right) \rho(\alpha)(a) \\
& =i_{X(b)} \circ d(\rho(\alpha))(a) \\
& =\left\langle\alpha,\left.\frac{d}{d t}\right|_{t=0} R\left(a+t X(b)_{a}\right)\right\rangle .
\end{aligned}
$$

Now, for any $Y_{a} \in T_{[a]} M C$, we have

$$
\begin{aligned}
\left.\frac{d}{d t}\right|_{t=0} R\left(a+t Y_{a}\right) & =1 \otimes Y_{a}+1 \otimes Y_{a} \otimes a+1 \otimes a \otimes Y_{a}+\cdots \\
& =\mathscr{B}\left(Y_{a}+\left(Y_{a} \otimes a\right)+\left(Y_{a} \otimes a \otimes a\right)+\cdots\right),
\end{aligned}
$$


where we used Connes' operator $B: \bar{C}_{\bullet}(A, A) \rightarrow \overline{\mathrm{CC}}_{\bullet}^{-}(A)$ from the long exact sequence (8) applied to $Y_{a}+\left(Y_{a} \otimes a\right)+\left(Y_{a} \otimes a \otimes a\right) \in \bar{C}_{\bullet}$ ( $(A, A)$. Thus, setting $Y_{a}=X(b)_{a}=d b+[a, b]$ in the above expression, we obtain

$$
\begin{aligned}
L_{X(b)} \rho(\alpha)(a)= & \langle\alpha, \mathcal{B}(d b+[a, b]+d b \otimes a+[a, b] \otimes a \\
& \quad+d b \otimes a \otimes a+[a, b] \otimes a \otimes a+\cdots)\rangle \\
= & \langle\alpha, \mathscr{B} \circ \delta(b+(b \otimes a)+(b \otimes a \otimes a)+\cdots)\rangle \\
= & \langle\alpha, \delta \circ \mathscr{B}(b+(b \otimes a)+(b \otimes a \otimes a)+\cdots)\rangle \\
= & \left\langle\delta^{*} \alpha, \mathcal{B}(b+(b \otimes a)+(b \otimes a \otimes a)+\cdots)\right\rangle \\
= & 0 .
\end{aligned}
$$

We are now ready to prove our main theorem.

Theorem 1. $\rho: \mathrm{HC}_{-}^{2 \bullet}(A) \rightarrow \mathcal{O}(\mathcal{M C})$ is a map of Lie algebras.

Proof. We saw in (13) that $\left.\frac{d}{d t}\right|_{t=0} R\left(a+t Y_{a}\right)=\mathscr{B}\left(Y_{a}+\left(Y_{a} \otimes a\right)+\cdots\right) \in \overline{\mathrm{CC}}_{\mathbf{0}}^{-}(A)$, where $\left(Y_{a}+\left(Y_{a} \otimes a\right)+\left(Y_{a} \otimes a \otimes a\right)+\cdots\right) \in \bar{C}_{\text {. }}(A, A)$ for $Y_{a} \in T_{[a]} \mathcal{M} C$. Therefore,

$$
\begin{aligned}
(d \rho(\alpha))_{a}\left(Y_{a}\right) & =\left\langle\alpha,\left.\frac{d}{d t}\right|_{t=0} R\left(a+t Y_{a}\right)\right\rangle \\
& =\left\langle\alpha, \mathscr{B}\left(Y_{a}+\left(Y_{a} \otimes a\right)+\left(Y_{a} \otimes a \otimes a\right)+\cdots\right)\right\rangle \\
& =\left\langle\mathscr{B}^{*} \alpha, Y_{a}+\left(Y_{a} \otimes a\right)+\left(Y_{a} \otimes a \otimes a\right)+\cdots\right\rangle \\
& =\left(\mathscr{B}^{*} \alpha\right)(1+a+a \otimes a+\cdots)\left(Y_{a}\right),
\end{aligned}
$$

where $\alpha \in \overline{\mathrm{CC}}_{-}^{\bullet}(A), \mathscr{B}^{*} \alpha \in \bar{C}^{\bullet}\left(A, A^{*}\right)$, and thus $\left(\mathscr{B}^{*} \alpha\right)\left(\sum_{i \geq 0} a^{\otimes i}\right) \in A^{*}$. Now, using the isomorphism $\omega_{\sharp}^{\bullet}: \mathrm{HH}^{\bullet}(A, A) \rightarrow \mathrm{HH}^{\bullet}\left(A, A^{*}\right)$ from Definition 4, we apply its inverse to obtain an element $\left[f_{\alpha}\right]:=\left(\omega_{\sharp}^{\bullet}\right)^{-1} \mathscr{B}^{\bullet}[\alpha] \in \operatorname{HH}^{\bullet}(A, A)$. We then claim that the Hamiltonian vector field $X_{a}^{\rho(\alpha)}$ may be expressed as

$$
X_{a}^{\rho(\alpha)}=f_{\alpha}\left(\sum_{i \geq 0} a^{\otimes i}\right) \in T_{[a]} \mathcal{M} C .
$$

This should be compared with [AZ], Lemma 7.2, and [Go], Proposition 3.7. To this end, first note that the relation $0=\left(\delta^{*} f\right)\left(\sum_{i \geq 0} a^{\otimes i}\right)=d_{a}\left(f\left(\sum_{i \geq 0} a^{\otimes i}\right)\right)$, for $f \in \bar{C}^{\bullet}(A, A)$, shows that $X_{a}^{\rho(\alpha)}$ given by equation (14) represents a well-defined class in $T_{[a]} \mathcal{M} \mathcal{C}$. We show (14) by applying the non-degeneracy of $\omega$ in the following equation, which is valid for any $Y_{a} \in T_{[a]} \mathcal{M} C$ :

$$
\begin{aligned}
\omega\left(f_{\alpha}\left(\sum a^{\otimes i}\right), Y_{a}\right) & =\operatorname{Tr}\left(f_{\alpha}\left(\sum a^{\otimes i}\right) \cdot Y_{a}\right) \\
& =\left(\omega_{\sharp} f_{\alpha}\right)\left(\sum a^{\otimes i}\right)\left(Y_{a}\right) \\
& =\left(\mathscr{B}^{*} \alpha\right)\left(\sum a^{\otimes i}\right)\left(Y_{a}\right) \\
& =(d \rho(\alpha))_{a}\left(Y_{a}\right) \\
& =\omega\left(X_{a}^{\rho(\alpha)}, Y_{a}\right) .
\end{aligned}
$$


Now, calculating the Lie bracket gives

$$
\begin{aligned}
\rho(\{\alpha, \beta\})(a) & =\langle\{[\alpha],[\beta]\},[R(a)]\rangle \\
& =\left\langle I^{\bullet}\left(\mathscr{B}^{\bullet}[\alpha] \sqcup \mathscr{B}^{\bullet}[\beta]\right),[R(a)]\right\rangle \\
& =\left\langle I^{\bullet} \omega_{\sharp}^{\bullet}\left(\left(\omega_{\sharp}^{\bullet}\right)^{-1} \mathscr{B}^{\bullet}[\alpha] \cup\left(\omega_{\sharp}^{\bullet}\right)^{-1} \mathscr{B}^{\bullet}[\beta]\right),[R(a)]\right\rangle \\
& =\left\langle\omega_{\sharp}^{\bullet}\left(\left[f_{\alpha}\right] \cup\left[f_{\beta}\right]\right), I \cdot[R(a)]\right\rangle \\
& =\left\langle\omega_{\sharp}^{*}\left(\left[f_{\alpha}\right] \cup\left[f_{\beta}\right]\right),[P(a)]\right\rangle .
\end{aligned}
$$

To evaluate this expression, note that for $f_{\alpha}: \bar{A}^{\otimes m} \rightarrow A$ and $f_{\beta}: \bar{A}^{\otimes n} \rightarrow A$, $\omega_{\sharp}^{*}\left(\left[f_{\alpha}\right] \cup\left[f_{\beta}\right]\right)$ is represented by the composition

$$
\bar{A}^{\otimes m+n} \stackrel{f_{\alpha} \otimes f_{\beta}}{\longrightarrow} A \otimes A \dot{\rightarrow} A \stackrel{\omega}{\rightarrow} A^{*} .
$$

The first arrow with $f_{\alpha} \otimes f_{\beta}$ applied to $P(a)=1+(1 \otimes a)+(1 \otimes a \otimes a)+\cdots \epsilon$ $\prod_{i \geq 0} A \otimes \bar{A}^{\otimes i}$ then gives an expression, where we apply $a$ to all possible inputs in $\bar{A}^{\otimes n+m}$. To this, we then apply the product in $A$, and apply $\omega$ with input $1 \in A$ since $P(a)=1 \otimes(\ldots)$. We thus obtain

$$
\begin{aligned}
\rho(\{\alpha, \beta\})(a) & =\operatorname{Tr}\left(f_{\alpha}(1+a+a \otimes a+\cdots) \cdot f_{\beta}(1+a+a \otimes a+\cdots) \cdot 1\right) \\
& \stackrel{(14)}{=} \operatorname{Tr}\left(X_{a}^{\rho(\alpha)} \cdot X_{a}^{\rho(\beta)}\right)=\omega\left(X_{a}^{\rho(\alpha)}, X_{a}^{\rho(\beta)}\right)=\{\rho(\alpha), \rho(\beta)\}(a) .
\end{aligned}
$$

This is the claim of the theorem.

\section{Comparison with generalized holonomy}

In this section we compare the map $\rho$ with the generalized holonomy map $\Psi$ studied in [AZ]. The relationship may be summarized in diagram (2). This shows how a special case the result of this paper relates to the main theorem of [AZ]. The map $\operatorname{Tr}: A \rightarrow \mathbb{C}$ is induced by the trace function on $\mathfrak{g} \subseteq \operatorname{GL}(n, \mathbb{C})$ and integration of forms on $M$; see Example 6.

Our model of $S^{1}$-equivariant de Rham forms of $L M$ is $(\Omega(L M)[u], d+u \Delta)$ where $u$ is a generator of degree 2 and $\Delta: \Omega^{\bullet}(L M) \rightarrow \Omega^{\bullet-1}(L M)$ is the map induced by the $S^{1}$-action on $L M$; see [GJP]. This model is quasi-isomorphic to the small Cartan model $\left(\Omega_{\text {inv }}(L M)[u], d+i_{X} u\right)$ for the $S^{1}$-action, where $X$ is the fundamental vector field generated by the natural action of $S^{1}$. The quasi-isomorphism is given by the averaging map $\Omega^{\bullet}(L M) \rightarrow \Omega_{\text {inv }}^{\bullet}(L M)$. More explicitly, for $\omega \in \Omega^{\bullet}(L M)$, $\Delta(\omega)$ is given by

$$
\Delta(\omega)=\int_{\text {fibre }} \operatorname{ev}^{*}(\omega) \in \Omega^{\bullet-1}(L M) .
$$




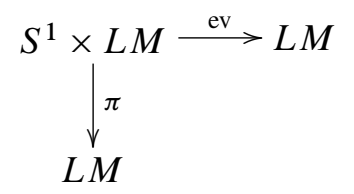

Chen's iterated integral map and the trace map on $g$ (see (6.3) [AZ], and Theorem A in [GJP]) yields a map, which we denote by

$$
S:\left(\bar{C}_{\bullet}(A, A), \delta\right) \rightarrow\left(\Omega^{\bullet}(L M), d\right) .
$$

The map $S$ induces the map $S^{\mathrm{HH}}:$ HH. $(A, A) \longrightarrow H^{\bullet}(L M)$ on homology, and, after applying the pairing between homology and cohomology groups, we get

$$
H .(L M) \stackrel{\sigma^{\mathrm{HH}}}{\longrightarrow} \mathrm{HH}^{\bullet}\left(A, A^{*}\right) .
$$

Extending $S$ by $u$-linearity, we obtain a map, which we denote by abuse of notation by the same letter,

$$
S:(\bar{C} \cdot(A, A)[u], \delta+u B) \rightarrow\left(\Omega^{\bullet}(L M)[u], d+u \Delta\right) .
$$

Since, by Lemma 3, $\left(\bar{C}_{\bullet}(A, A)[u], \delta+u B\right)$ and $\left(\bar{C}_{\bullet}(A, A)[[u]], \delta+u B\right)$ are quasiisomorphic in our setting, we obtain the induced map $S^{\mathrm{HC}}: \mathrm{HC}_{\bullet}^{-}(A) \longrightarrow H_{S^{1}}^{\bullet^{\prime}}(L M)$ on homology. Composing $S^{\mathrm{HC}}$ with the map $R: M C \rightarrow \overline{\mathrm{CC}}_{\bullet}^{-}(A)=\bar{C}_{\bullet}(A, A)[u]$ from Section 4, we get

$$
M C \stackrel{R}{\rightarrow} \mathrm{HC}_{\bullet}^{-}(A) \stackrel{S^{\mathrm{HC}}}{\longrightarrow} H_{S^{1}}^{\cdot}(L M) .
$$

Thus by duality, and using Lemma 9, we have

$$
H_{\bullet}^{S^{1}}(L M) \stackrel{\sigma=\sigma^{\mathrm{HC}}}{\longrightarrow} \mathrm{HC}_{-}^{\bullet}(A) \stackrel{\rho}{\rightarrow} \mathcal{O}(\mathcal{M} C) .
$$

The composition $\rho \circ \sigma$ is the generalized holonomy map $\Psi$ discussed in [AZ].

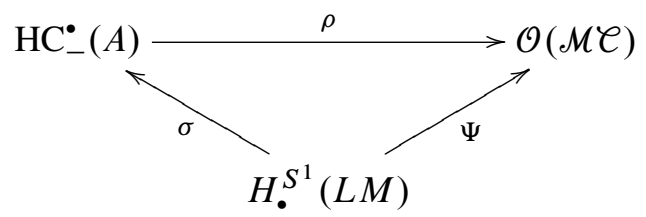

It was proved in [AZ] that $\Psi$ is the morphism of Lie algebras. We will shortly see how this is a consequence of Theorem 1 . We first recall the following theorem.

Theorem 10 (S. Merkulov [Mer]). The Chen integral induces a map of algebras $(H .(L M), \bullet) \rightarrow\left(\mathrm{HH}^{\bullet}(A, A), \cup\right)$. 
Thus, by definition, $\sigma^{\mathrm{HH}}:\left(H_{\bullet}(L M), \bullet\right) \rightarrow\left(\mathrm{HH}^{\bullet}\left(A, A^{*}\right), \sqcup\right)$ is also a map of algebras. With this, we can now prove the following statement.

Theorem 11. The map $\sigma:\left(H_{\bullet}^{S^{1}}(L M),\{\cdot, \cdot\}\right) \rightarrow\left(\mathrm{HC}_{-}^{\bullet}(A),\{\cdot, \cdot\}\right)$ induced by the Chen iterated integrals is a map of Lie algebras. Here, the first bracket is the string bracket and the second one is defined in the statement of Proposition 5.

Proof. The brackets on $H_{\bullet}^{S^{1}}(L M)$ and $\mathrm{HC}_{-}^{\bullet}(A)$ are determined by the products on $H_{\bullet}(L M)$ and $\mathrm{HC}^{\bullet}\left(A, A^{*}\right)$, together with the maps in the corresponding Gysin long exact sequences. By Theorem 10, it thus remains to show that the long exact sequences correspond to each other, i.e., that the following diagrams commute:

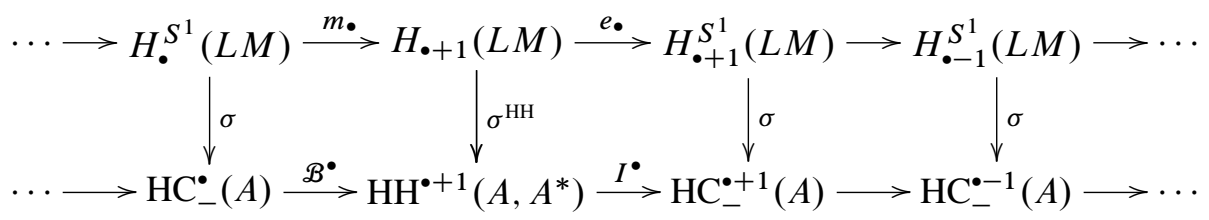

Equivalently, we need to show the commutativity of the dual sequence:

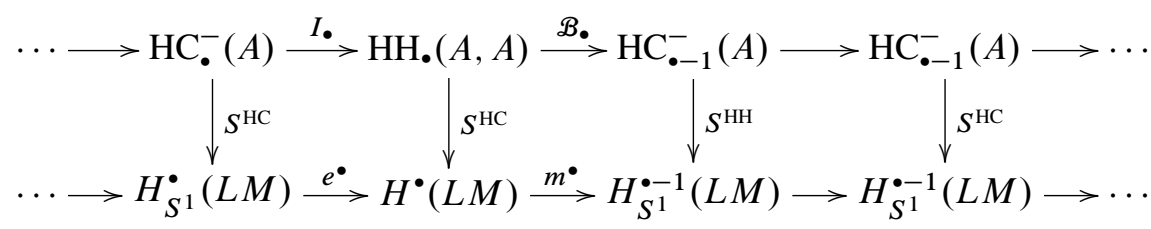

The top long exact sequence is induced by the short exact sequence (7), while the bottom one is induced by the short exact sequence

$$
0 \rightarrow\left(\Omega^{\bullet}(M)[u], d+u \Delta\right) \stackrel{\times u}{\longrightarrow}\left(\Omega^{\bullet}(M)[u], d+u \Delta\right) \stackrel{j}{\rightarrow} \Omega^{\bullet}(M) \rightarrow 0,
$$

where $j\left(\sum a_{i} u^{i}\right)=a_{0}$, cf. [GS], [Ma]. In this picture, $m^{\bullet}$ corresponds to the connecting map of the long exact sequence (17). By a diagram chasing argument one finds that $m^{\bullet}=(i \circ \Delta)^{\bullet}$, where $i: \Omega^{\bullet}(M) \hookrightarrow \Omega^{\bullet}(M)[u]$ corresponds to $\mathcal{B}^{\bullet}=$ (inc $\circ B)^{\bullet}$ using Chen iterated integrals as corollary of Theorem A in [GJP]. Note that $i$ is not a chain map, whereas $i \circ \Delta$ is a chain map since $\Delta d=d \Delta$ and $\Delta^{2}=0$, (cf. [GJP]).

\section{6. $A_{\infty}$-generalization}

The previous sections, dealing with the case of dgas $(A, d, \cdot)$ with invariant inner product $\omega: A \otimes A \rightarrow k$, generalize in a straightforward way to the setting of cyclic $A_{\infty}$-algebras. In this section, we recall the relevant definitions (cf. [T]), and adopt the above to this situation. 
Definition 12. An $A_{\infty}$-algebra on $A$ consists of a sequence of maps $\left\{\mu_{n}\right\}_{n \geq 1}$, where $\mu_{n}: A^{\otimes n} \rightarrow A$ is of degree $(2-n)$, such that, for all $n \geq 1$,

$$
\sum_{\substack{k+l=n+1 \\ r=0, \ldots, n-l}}(-1)^{\epsilon_{l}^{r}} \cdot \mu_{k}\left(a_{1} \otimes \cdots \otimes \mu_{l}\left(a_{r+1} \otimes \cdots \otimes a_{r+l}\right) \otimes \cdots \otimes a_{n}\right)=0,
$$

where $\epsilon_{l}^{r}=(l-1) \cdot\left(\left|a_{1}\right|+\cdots+\left|a_{r}\right|-r\right)$. A unit is an element $1 \in k \subset A^{0}$ such that $\mu_{2}(a, 1)=\mu_{2}(1, a)=a$, and $\mu_{n}(\cdots \otimes 1 \otimes \ldots)=0$ for $n \neq 2$. Again, we write $\bar{A}=A / k$. We define the Hochschild chain complex of $A$ with values in $A$ or $A^{*}$ to be the vector spaces $\bar{C}_{\bullet}(A, A)$ and $\bar{C}_{\bullet}\left(A, A^{*}\right)$ from equation (3) with the differentials modified as follows:

$$
\begin{aligned}
& \delta: \bar{C}_{\bullet}(A, A) \rightarrow \bar{C}_{\bullet}(A, A), \delta\left(a_{0} \otimes \cdots \otimes a_{n}\right) \\
& =\sum \pm a_{0} \otimes \cdots \otimes \mu_{k}(\ldots) \otimes \cdots \otimes a_{n} \\
& +\sum \pm \mu_{k}\left(a_{s} \otimes \cdots \otimes a_{0} \otimes \cdots \otimes a_{r}\right) \otimes a_{r+1} \otimes \cdots \otimes a_{s-1}, \\
& \delta: \bar{C}_{\bullet}\left(A, A^{*}\right) \rightarrow \bar{C}_{\bullet}\left(A, A^{*}\right), \delta\left(a_{0}^{*} \otimes \cdots \otimes a_{n}\right) \\
& =\sum \pm a_{0}^{*} \otimes \cdots \otimes \mu_{k}(\ldots) \otimes \cdots \otimes a_{n} \\
& +\sum \pm \mu_{k}^{*}\left(a_{s} \otimes \cdots \otimes a_{0}^{*} \otimes \cdots \otimes a_{r}\right) \otimes a_{r+1} \otimes \cdots \otimes a_{s-1},
\end{aligned}
$$

where $\mu_{k}^{*}\left(a_{s} \otimes \cdots \otimes a_{0}^{*} \otimes \cdots \otimes a_{r}\right) \in A^{*}$ is given by

$\mu_{k}^{*}\left(a_{s} \otimes \cdots \otimes a_{n} \otimes a_{0}^{*} \otimes a_{1} \otimes \cdots \otimes a_{r}\right)(a):= \pm a_{0}^{*}\left(\mu_{k}\left(a_{1} \otimes \cdots \otimes a_{r} \otimes a \otimes a_{s} \otimes \cdots \otimes a_{n}\right)\right)$.

Here, the signs are given by the usual Koszul rule, where a factor of $(-1)^{\epsilon \epsilon^{\prime}}$ is introduced whenever elements of degree $\epsilon$ and $\epsilon^{\prime}$ are being commuted. For an explicit discussion of the signs; see e.g. [T]. Similarly, $\bar{C}^{\bullet}(A, A)$ and $\bar{C}^{\bullet}\left(A, A^{*}\right)$ are defined by the spaces from (4) with the modified differentials

$$
\begin{gathered}
\delta^{*}: \bar{C}^{\bullet}(A, A) \rightarrow \bar{C}^{\bullet}(A, A), \\
\delta^{*} f\left(a_{1} \otimes \cdots \otimes a_{n}\right)=\sum \pm f\left(a_{1} \otimes \cdots \otimes \mu_{k}(\ldots) \otimes \cdots \otimes a_{n}\right) \\
+\sum \pm \mu_{k}\left(a_{1} \otimes \cdots \otimes f(\ldots) \otimes \cdots \otimes a_{n}\right), \\
\delta^{*}: \bar{C}^{\bullet}\left(A, A^{*}\right) \rightarrow \bar{C}^{\bullet}\left(A, A^{*}\right), \\
\delta^{*} f\left(a_{1} \otimes \cdots \otimes a_{n}\right)=\sum \pm f\left(a_{1} \otimes \cdots \otimes \mu_{k}(\ldots) \otimes \cdots \otimes a_{n}\right) \\
+\sum \pm \mu_{k}^{*}\left(a_{1} \otimes \cdots \otimes f(\ldots) \otimes \cdots \otimes a_{n}\right) .
\end{gathered}
$$

Since $\delta^{2}=0,\left(\delta^{*}\right)^{2}=0$ in all the above cases, we obtain the associated homologies and cohomologies $H_{\bullet}(A, A), H_{\bullet}\left(A, A^{*}\right), H^{\bullet}(A, A)$, and $H^{\bullet}\left(A, A^{*}\right)$.

There is a generalized cup product $\cup$ on $H^{\bullet}(A, A)$ induced by $(f \cup g)\left(a_{1} \otimes \cdots \otimes a_{n}\right):=\sum_{k \geq 2} \pm \mu_{k}\left(a_{1} \otimes \cdots \otimes f(\ldots) \otimes \cdots \otimes g(\ldots) \otimes \cdots \otimes a_{n}\right)$.

Furthermore, equation (5) defines an operator $B: \bar{C}_{.}(A, A) \rightarrow \bar{C}_{\text {. }}(A, A)$ with $B^{2}=$ $\delta B+B \delta=0$. We define the negative cyclic chains $\overline{\mathrm{CC}}_{\bullet}^{-}(A)$ of $A$ to be the vector space 
$\bar{C}_{\bullet}(A, A)[[u]]$ with differential $\delta+u B$, and denote the negative cyclic homology by $\mathrm{HC}_{\bullet}^{-}(A)$. Dualizing $\overline{\mathrm{CC}}_{\bullet}^{-}(A)$, we obtain $\overline{\mathrm{CC}}_{-}^{\bullet}(A)$ with dual differential and denote the negative cyclic cohomology by $\mathrm{HC}_{-}^{\bullet}(A)$. For the same reasons as in Section 2, we obtain the long exact sequences (8) and (9).

Finally, assume that we have a trace $\operatorname{Tr}: A \rightarrow k$ such that the associated map $\omega: A \otimes A \rightarrow k, \omega(a, b)=\operatorname{Tr}\left(\mu_{2}(a \otimes b)\right)$ is a quasi-isomorphism, which satisfies

$$
\omega\left(\mu_{n}\left(a_{1} \otimes \cdots \otimes a_{n}\right), a_{n+1}\right)= \pm \omega\left(\mu_{n}\left(a_{n+1} \otimes a_{1} \otimes \cdots \otimes a_{n-1}\right), a_{n}\right),
$$

for $n \geq 1$. In this case, $\omega: A \rightarrow A^{*}$ induces a map of the Hochschild cohomologies $H^{\bullet}(A, A) \rightarrow H^{\bullet}\left(A, A^{*}\right), \omega_{\sharp}^{\bullet}(f)=\omega \circ f$, which we assume to be an isomorphism. Thus, we may transfer the product $\cup$ on $H^{\bullet}(A, A)$ to a product $\sqcup$ on $H^{\bullet}\left(A, A^{*}\right)$. $\left(\mathrm{HH}^{\bullet}\left(A, A^{*}\right), \sqcup, \Delta=B^{*}\right)$ is a BV-algebra, cf. [T], so that we obtain the Lie bracket $\{a, b\}:=I^{\bullet}\left(\mathscr{B}^{\bullet}(a) \sqcup \mathscr{B}^{\bullet}(b)\right)$ on $\mathrm{HC}_{-}^{\bullet}(A)$ just as in Proposition 5.

Using this setup, we may now also generalize Section 3.

Definition 13. Recall that there are maps from the $n$-th symmetric power of a vector space to the $n$-th tensor power $S^{n}: A^{\wedge n} \rightarrow A^{\otimes n}$, where $S^{n}\left(a_{1} \wedge \cdots \wedge a_{n}\right)=$ $\sum_{\sigma \in \Sigma_{n}}(-1)^{\epsilon \sigma}\left(a_{\sigma(1)} \otimes \cdots \otimes a_{\sigma(n)}\right)$. Defining $v_{n}: A^{\wedge n} \rightarrow A$ as $v_{n}:=\mu_{n} \circ S^{n}$, we obtain an $L_{\infty}$-algebra on $A$, cf. [LM], Theorem 3.1. Furthermore, from (18), it is immediate to see that, for $n \geq 1$, we have

$$
\omega\left(v_{n}\left(a_{1} \wedge \cdots \wedge a_{n}\right), a_{n+1}\right)= \pm \cdot \omega\left(v_{n}\left(a_{n+1} \wedge a_{1} \wedge \cdots \wedge a_{n-1}\right), a_{n}\right) .
$$

For this $L_{\infty}$-algebra, recall from [GG], Section 2, that the Maurer-Cartan solutions are defined by

$$
\begin{aligned}
& M C:=\left\{a \in A^{\text {odd }} \mid \nu_{1}(a)+\frac{1}{2 !} \nu_{2}(a \wedge a)+\frac{1}{3 !} \nu_{3}(a \wedge a \wedge a)+\cdots=0\right\}, \\
& \mathcal{M C}:=M C / \sim,
\end{aligned}
$$

where the equivalence is again generated by the infinitesimal action of $A^{\text {even }}$ on $A^{\text {odd }}$ and where, for $a \in A^{\text {even }}$, the vector field $\xi_{x}$ on $A^{\text {odd }}$ is defined by

$$
\xi_{x}(a)=v_{1}(x)+v_{2}(a \wedge x)+\frac{1}{2 !} v_{3}(a \wedge a \wedge x)+\cdots .
$$

Note that under the above assumptions the tangent space $T_{[a]} \mathcal{M} \mathcal{C}$ to $\mathcal{M} \mathcal{C}$ at $[a]$ is the self-dual 3-term complex

$$
T_{[a]}^{-1} \mathcal{M} \mathcal{M}:=A^{\text {even }} \stackrel{\xi(a)}{\longrightarrow} T_{[a]}^{0} \mathcal{M} \mathcal{M}:=T_{a} A^{\text {odd }}=A^{\text {odd }} \stackrel{\mu_{a}^{\prime}}{\longrightarrow} T_{[a]}^{1} \mathcal{M} \mathcal{M}:=A^{\text {even} *},
$$

where

$$
\mu_{a}^{\prime}(b)=v_{1}(b)+v_{2}(a \wedge b)+\frac{1}{2 !} v_{3}(a \wedge a \wedge b)+\cdots
$$


The self-duality at the middle term is given by the symplectic form

$$
\omega\left(X_{a}, Y_{a}\right)=\operatorname{Tr}\left(\mu_{2}\left(X_{a} \otimes Y_{a}\right)\right) \in k .
$$

This can be used to define the Hamiltonian vector field $X^{\psi}$ associated to a function $\psi \in \mathcal{O}(\mathcal{M C})$, and thus the Lie bracket on $\mathcal{O}(\mathcal{M C})$ via the usual formula $\{\psi, \chi\}=$ $\omega\left(X^{\psi}, X^{\chi}\right)$.

We may now define the map $P: M C \rightarrow \bar{C}_{\text {. }}(A, A)$ by

$$
P(a):=\sum_{i \geq 0} 1 \otimes a^{\otimes i}=\left(1 \otimes 1_{\bar{A} \otimes 0}\right)+(1 \otimes a)+(1 \otimes a \otimes a)+\cdots,
$$

and $R=i n c \circ P: M C \rightarrow \overline{\mathrm{CC}}^{-}(A)$. As in Definition 8, we may again see that $\delta(P(a))=0$, and $(\delta+u B)(R(a))=0$, and we define

$$
\rho: \operatorname{HC}_{-}^{2 \bullet}(A) \rightarrow \mathcal{O}(\mathcal{M} C), \quad \rho([\alpha])([a]):=\langle[\alpha],[R(a)]\rangle=\langle\alpha, R(a)\rangle,
$$

for $[\alpha] \in \mathrm{HC}^{\bullet}(A),[a] \in \mathcal{M} \mathcal{C}$. With this, we have the same theorem as in the previous sections.

Theorem 14. The map $\rho$ is a well-defined map of Lie algebras.

\section{References}

[AZ] H. Abbaspour and M. Zeinalian, String bracket and flat connections. Algebr. Geom. Topol. 7 (2007), 197-231. Zbl 1142.55006 MR 2308942

[AB] M. F. Atiyah and R. Bott, The moment map and equivariant cohomology. Topology 23 (1984), 1-28. Zbl 0521.58025 MR 721448

[CCR] A. S. Cattaneo, P. Cotta-Ramusino, and M. Rinaldi, Loop and path spaces and fourdimensional $B F$ theories: connections, holonomies and observables. Comm. Math. Phys. 204 (1999), 493-524. Zbl 0939.58009 MR 1707635

[CFP] A. S. Cattaneo, J. Fröhlich, and B. Pedrini, Topological field theory interpretation of string topology. Comm. Math. Phys. 240 (2003), 397-421. Zbl 1052.81095 MR 2005849

[CR] A. S. Cattaneo and C. A. Rossi, Higher-dimensional $B F$ theories in the BatalinVilkovisky formalism: the BV action and generalized Wilson loops. Comm. Math. Phys. 221 (2001), 591-657. Zbl 1009.81071 MR 1852055

[CS] M. Chas and D. Sullivan, String topology. Ann. of Math. to appear. arXiv:math/9911159

[C] K. Costello, Topological conformal field theories and gauge theories. Geom. Topol. 11 (2007), 1539-1579. Zbl 1139.32006 MR 2326951

[CTZ] K. Costello, T. Tradler, and M. Zeinalian, Closed string TCFT for Hermitian CalabiYau alliptic spaces. Preprint 2008. arXiv:0807.3052 
[DT] S. K. Donaldson and R. P. Thomas, Gauge theory in higher dimensions. In The geometric universe (Oxford, 1996), Oxford University Press, Oxford 1998, 31-47. Zbl 0926.58003 MR 1634503

[GG] W. L. Gan and V. Ginzburg, Hamiltonian reduction and Maurer-Cartan equations. Moscow Math. J. 4 (2004), 719-727. Zbl 1080.53083 MR 2119146

[GJP] E. Getzler, J. D. S. Jones, and S. Petrack, Differential forms on loop spaces and the cyclic bar complex. Topology 30 (1991), 339-371. Zbl 0729.58004 MR 1113683

[Gi] P. B. Gilkey, The index theorem and the heat equation. Publish or Perish, Boston 1974. Zbl 0287.58006 MR 0458504

[Go] W. M. Goldman, Invariant functions on Lie groups and Hamiltonian flows of surface group representations. Invent. Math. 85 (1986), 263-302.Zbl 0619.58021 MR 846929

[GKM] M. Goresky, R. Kottwitz, and R. MacPherson, Equivariant cohomology, Koszul duality, and the localization theorem. Invent. Math. 131 (1998), 25-83. Zbl 0897.22009 MR 1489894

[GS] V. W. Guillemin and S. Sternberg, Supersymmetry and equivariant de Rham theory. Mathematics Past and Present, Springer-Verlag, Berlin 1999. Zbl 0934.55007 MR 1689252

[HL] A. Hamilton and A. Lazarev, Symplectic $A_{\infty}$ algebras and string topology operations. In Geometry, topology, and mathematical physics, Amer. Math. Soc. Transl. Ser. (2) 224, Amer. Math. Soc., Providence, RI, 2008, 147-157. Zbl 1153.55009 MR2462359

[J] J. D. S. Jones, Cyclic homology and equivariant homology. Invent. Math. 87 (1987), 403-423. Zbl 0644.55005 MR 870737

[K] S. Kobayashi, Differential geometry of complex vector bundles. Kanô Memorial Lectures 5, Publ. Math. Soc. Japan 15, Iwanami Shoten Publishers, Tokyo; Princeton University Press, Princeton, NJ, 1987. Zbl 0708.53002 MR 0909698

[LM] T. Lada and M. Markl, Strongly homotopy Lie algebras. Comm. Algebra 23 (1995), 2147-2161. Zbl 0999.17019 MR 1327129

[L] J.-L. Loday, Cyclic homology. 2nd ed., Grundl. Math. Wiss. 301, Springer-Verlag, Berlin 1998. Zbl 0885.18007 MR 1600246

[Ma] P. Manoharan, The equivariant de Rham theorem on Fréchet $S^{1}$-manifolds. Ann. Global Anal. Geom. 11 (1993), 119-123. Zbl 0811.58010 MR 1225432

[Men] L. Menichi, Batalin-Vilkovisky algebras and cyclic cohomology of Hopf algebras. K-Theory 32 (2004), 231-251. Zbl 1101.19003 MR 2114167

[Mer] S. A. Merkulov, De Rham model for string topology. Internat. Math. Res. Notices 2004 (2004), 2955-2981. Zbl 1066.55008 MR 2099178

[T] T. Tradler, The BV-algebra on Hochschild cohomology induced by infinity inner products. Ann. Inst. Fourier (Grenoble) to appear.

[TZ] T. Tradler and M. Zeinalian, Algebraic string operations. K-Theory 38 (2007), 59-82. Zbl 1144.55012 MR 2353864

Received January 28, 2009 
H. Abbaspour, Laboratoire de Mathématiques Jean Leray, Université de Nantes,

2, rue de la Houssinière, BP 92208, 44322 Nantes Cedex 3, France

E-mail: abbaspour@univ-nantes.fr

T. Tradler, Department of Mathematics, New York City College of Technology, 300 Jay Street, Brooklyn, NY 11201, U.S.A.

E-mail: ttradler@citytech.cuny.edu

M. Zeinalian, Department of Mathematics, Long Island University, C.W. Post College, Brookville, NY 11548, U.S.A.

E-mail: mzeinalian@liu.edu 